\title{
Large anomalous Hall effect in a non-collinear antiferromagnet at room temperature
}

\author{
S. Nakatsuji* , N. Kiyohara \& T. Higo
}

\section{PAPER ABSTRACT}

In ferromagnetic conductors, an electric current may induce a transverse voltage drop in zero applied magnetic field: this anomalous Hall effect is observed to be proportional to magnetization, and thus is not usually seen in antiferromagnets in zero field. Recent developments in theory and experiment have provided a framework for understanding the anomalous Hall effect using Berry-phase concepts, and this perspective has led to predictions that, under certain conditions, a large anomalous Hall effect may appear in spin liquids and antiferromagnets without net spin magnetization. Although such a spontaneous Hall effect has now been observed in a spin liquid state, a zero-field anomalous Hall effect has hitherto not been reported for antiferromagnets. Here we report empirical evidence for a large anomalous Hall effect in an antiferromagnet that has vanishingly small magnetization. In particular, we find that $\mathrm{Mn}_{3} \mathrm{Sn}$, an antiferromagnet that has a non-collinear 120-degree spin order, exhibits a large anomalous Hall conductivity of around 20 per ohm per centimetre at room temperature and more than 100 per ohm per centimetre at low temperatures, reaching the same order of magnitude as in ferromagnetic metals. Notably, the chiral antiferromagnetic state has a very weak and soft ferromagnetic moment of about 0.002 Bohr magnetons per Mn atom, allowing us to switch the sign of the Hall effect with a small magnetic field of around a few hundred oersted. This soft response of the large anomalous Hall effect could be useful for various applications including spintronics-for example, to develop a memory device that produces almost no perturbing stray fields.

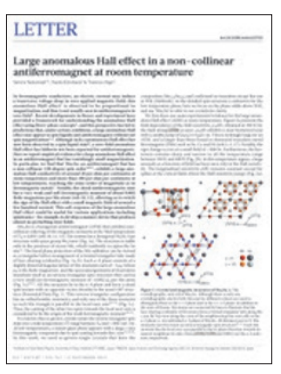

\section{SUMMARY}

Using spin in an antiferromagnet for data processing and storage. In the field of spintronics, the goal is to use electron spin, rather than charge, to control and store information. An example of such control is the anomalous Hall effect, which is usually observed in ordinary ferromagnets, such as iron or nickel. This effect has now been reported in an antiferromagnet, despite its almost zero magnetization, paving the way to nextgeneration memory storage and ultrafast data processing.

\section{The problem}

Metal ferromagnets, including iron, cobalt and nickel, are often used in spintronic devices because they retain their magnetism easily and their ordered parallel magnetic spins are useful for processing and storing data. Antiferromagnetic materials also have ordered spin states, but their spins are often aligned antiparallel with their neighbours, resulting in no overall magnetization. Such spin states are difficult to control, making writing, reading and storing magnetic data seemingly impossible.

A well-known feature of ferromagnets is the anomalous Hall effect $(\mathrm{AHE})^{1}$, which arises in ferromagnets even without an external field (Graphical abstract, a). Since its discovery by Edwin Hall in 1880, the AHE has been thought to be proportional to magnetization, so it would not be observed in antiferromagnets. But it is now understood that it may theoretically arise even in magnets with zero magnetization, including antiferromagnets ${ }^{2-4}$ and spin liquids. Indeed, a spontaneous Hall effect has recently been found ${ }^{5}$ in the spin liquid state of $\operatorname{Pr}_{2} \operatorname{Ir}_{2} \mathrm{O}_{7}$. However, no one has yet observed the AHE in an antiferromagnet.

\section{The solution}

Here we report that the antiferromagnet $\mathrm{Mn}_{3} \mathrm{Sn}$ can demonstrate an AHE that is even larger than those seen in ordinary ferromagnets. We focused on $\mathrm{Mn}_{3} \mathrm{Sn}$, which has a geometrically frustrated kagome lattice supporting an inverse triangular spin structure (Graphical abstract, b). We measured the Hall effect using single crystals, which are easily made and are safe and stable in air. We found that it exhibits AHE at room temperature and can be switched by a weak magnetic field. Our discovery indicates that the AHE arises not from the internal magnetic field due to magnetization, as in ferromagnets, but from the fictitious field' induced by a non-trivial topology of electronic band structure. Furthermore, a tiny magnetic field of a few hundred oersted is sufficient to switch the direction of the spin state in the antiferromagnet and hence to change the orientation of the large fictitious field of more than a few hundred tesla.

doi: 10.1038 /nature 15723

- RECEIVED 3 May 2015

- ACCEPTED 1 September 2015

- PUBLISHED ONLINE 28 October 2015

\section{The implications}

The exceptionally large $\mathrm{AHE}$ of $\mathrm{Mn}_{3} \mathrm{Sn}$ has several applications. 
Researchers have been searching for useful antiferromagnetic materials to develop the next generation of memory devices. Unlike the ferromagnets that are typically used for spintronics, antiferromagnets produce almost no stray fields of their own and therefore do not disturb the neighbouring memory cells. As ferromagnetic memory devices are reaching the limits of miniaturization, the use of antiferromagnets would allow for even higher storage density. Moreover, antiferromagnets have much faster spin dynamics than ferromagnets, making them ideal for ultrafast data processing.

So far it has been difficult to write information using antiferromagnets because it is not easy to manipulate magnetic domains that have vanishingly small magnetization. In addition, even if the domains were controlled, the macroscopic responses of an antiferromagnet are often so weak that it would be extremely hard to read the information.

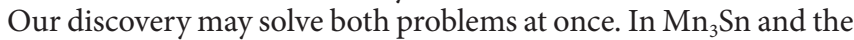
related $\mathrm{Mn}_{3} \mathrm{Ge}^{6,7}$, the spin state can be switched by applying a small field of only a few hundred oersted. At the same time, these materials generate a large and easily detectable change in the Hall voltage. Our discovery therefore paves the way for the development of non-volatile memory and high-speed switching devices based on antiferromagnets.

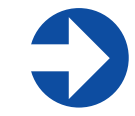

The full version of this article is at Nature's website at go.nature.com/29nwwlj

\section{GRAPHICAL ABSTRACT}

a
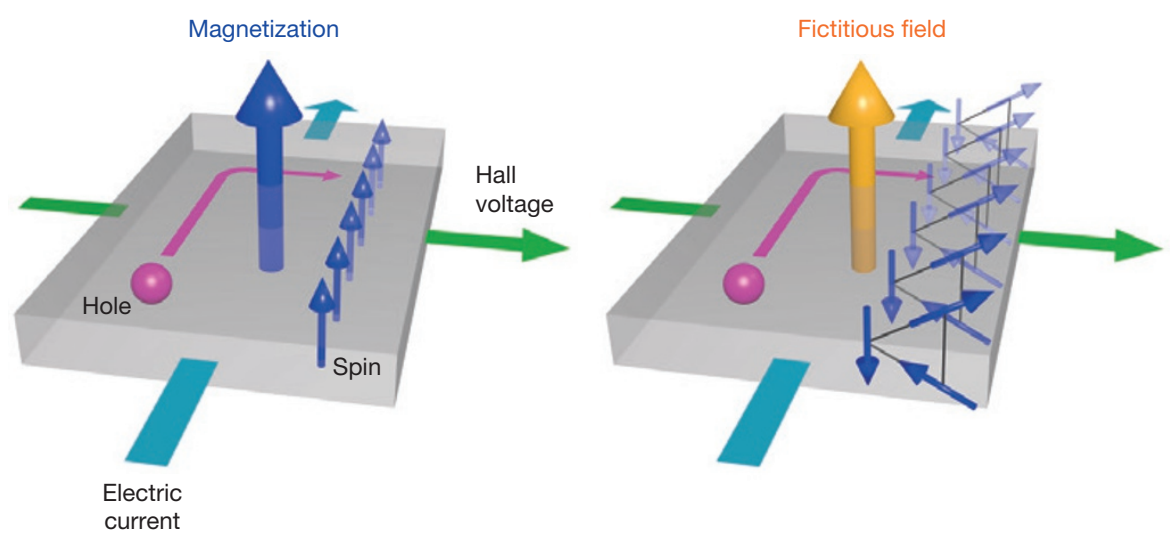

b

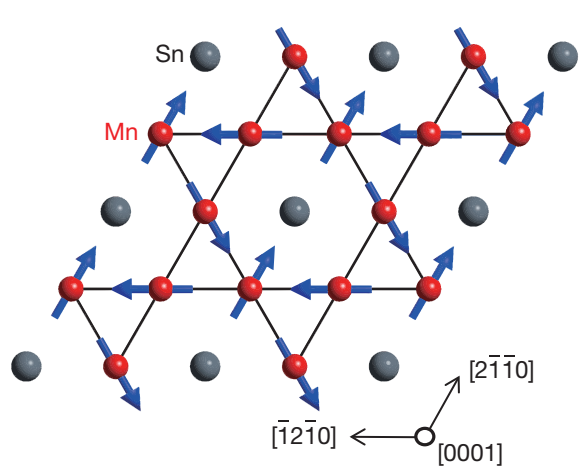

C

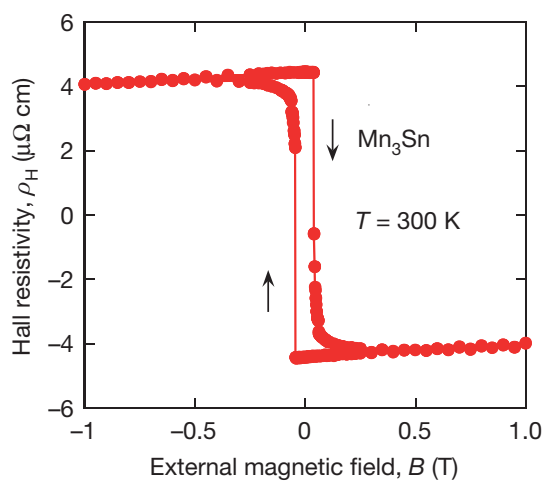

Magnetic behaviour and crystal structure of $\mathrm{Mn}_{3} \mathrm{Sn}$. a, Anomalous Hall effect in ferromagnets (left) and in antiferromagnets (right) induced by bending the trajectory of conducting carriers (in this case holes) by magnetization and fictitious field, respectively. In ferromagnets, a spontaneous Hall effect appears at zero field, which is proportional to magnetization. In antiferromagnets, an anomalous Hall effect is driven by the fictitious field induced by the non-trivial topology of electronic band structure. b, Crystal and spin structure of $\mathrm{Mn}_{3} \mathrm{Sn}$. Each $\mathrm{Mn}$ has a magnetic moment (arrow). An inverse triangular spin structure appears because of the geometrical frustration resulting from the kagome lattice formed by Mn. c, Magnetic field dependence on the Hall resistivity in $\mathrm{Mn}_{3} \mathrm{Sn}$ at room temperature.

\section{FURTHER READING}

1. Nagaosa, N., Sinova, J., Onoda, S., MacDonald, A. H. \& Ong, N. P. Anomalous Hall effect. Rev. Mod. Phys. 82, 1539-1592 (2010).

This paper provides a comprehensive review of recent theoretical and experimental work on the AHE in ferromagnets.

2. Shindou, R. \& Nagaosa, N. Orbital ferromagnetism and anomalous Hall effect in antiferromagnets on the distorted fcc lattice. Phys. Rev. Lett. 87, 116801 (2001). An early theoretical work on the AHE in a geometrically frustrated antiferromagnet with a non-coplanar spin structure.

3. Chen, H., Niu, Q. \& MacDonald, A. H. Anomalous Hall effect arising from noncollinear antiferromagnetism. Phys. Rev. Lett. 112, 017205 (2014).

A theoretical work on the AHE that may arise in a non-collinear antiferromagnet with a frustrated kagome lattice.

4. Kübler, J. \& Felser, C. Non-collinear antiferromagnets and the anomalous Hall effect. Europhys. Lett. 108, 67001 (2014). Theoretical work that found the possible AHE in the antiferromagnets $\mathrm{Mn}_{3} \mathrm{Sn}$ and $\mathrm{Mn}_{3} \mathrm{Ge}$.

5. Machida, Y., Nakatsuji, S., Onoda, S., Tayama, T. \& Sakakibara, T. Time-reversal symmetry breaking and spontaneous Hall effect without magnetic dipole order. Nature 463, 210-213 (2010).

This paper reports the experimental observation of a large AHE in the spin liquid state of the spin ice compound $\operatorname{Pr}_{2} \mathrm{Ir}_{2} \mathrm{O}_{7}$.

6. Kiyohara, N., Tomita, T. \& Nakatsuji, S. Giant anomalous Hall effect in the chira antiferromagnet $\mathrm{Mn}_{3} \mathrm{Ge}$. Phys. Rev. Appl. 5, 064009 (2016).

This reports the experimental observation of a very large AHE in the non-collinear antiferromagnet $\mathrm{Mn}_{3} \mathrm{Ge}$ at room temperature.

7. Nayak, A. K. et al. Large anomalous Hall effect driven by a nonvanishing Berry curvature in the noncolinear antiferromagnet $\mathrm{Mn}_{3} \mathrm{Ge}$. Sci. Adv. 2, e1501870 (2016). This reports the experimental observation of the large AHE and the theoretical calculation on the spin Hall effect in $\mathrm{Mn}_{3} \mathrm{Ge}$.

\section{RELATED CONTENT}

Antiferromagnetic spintronics

T. Jungwirth, X. Marti, P. Wadley \& J. Wunderlich go.nature.com/29xvgh7

Introduction to Frustrated Magnetism

Claudine Lacroix, Philippe Mendels \& Frédéric

Mila (eds)

go.nature.com/29w06b0

Correlated quantum phenomena in the strong spin-orbit regime

William Witczak-Krempa, Gang Chen, Yong Baek Kim \& Leon Balents

go.nature.com/2aeryza 